Supplementary information for

\title{
Biocatalytic fabrication of $\alpha$-glucan-coated porous starch granules by amylolytic and glucan-synthesizing enzymes as a target-specific delivery carrier
}

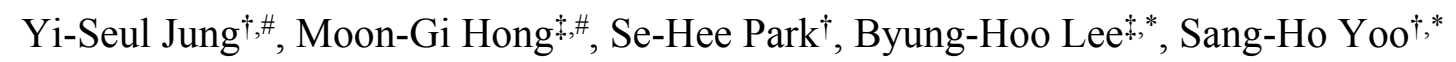

$\dagger^{\dagger}$ Department of Food Science \& Biotechnology and Carbohydrate Bioproduct Research Center, Sejong University, Seoul 05006, Republic of Korea

Department of Food Science \& Biotechnology, Gachon University, Seongnam 13120, Republic of Korea

\#These authors contributed equally

*Corresponding authors:

Byung-Hoo Lee (Tel.: +82 31-750-5405, e-mail address: blee@gachon.ac.kr)

Sang-Ho Yoo (Tel.: +82 2-3408-3221, e-mail address: shyoo@sejong.ac.kr) 

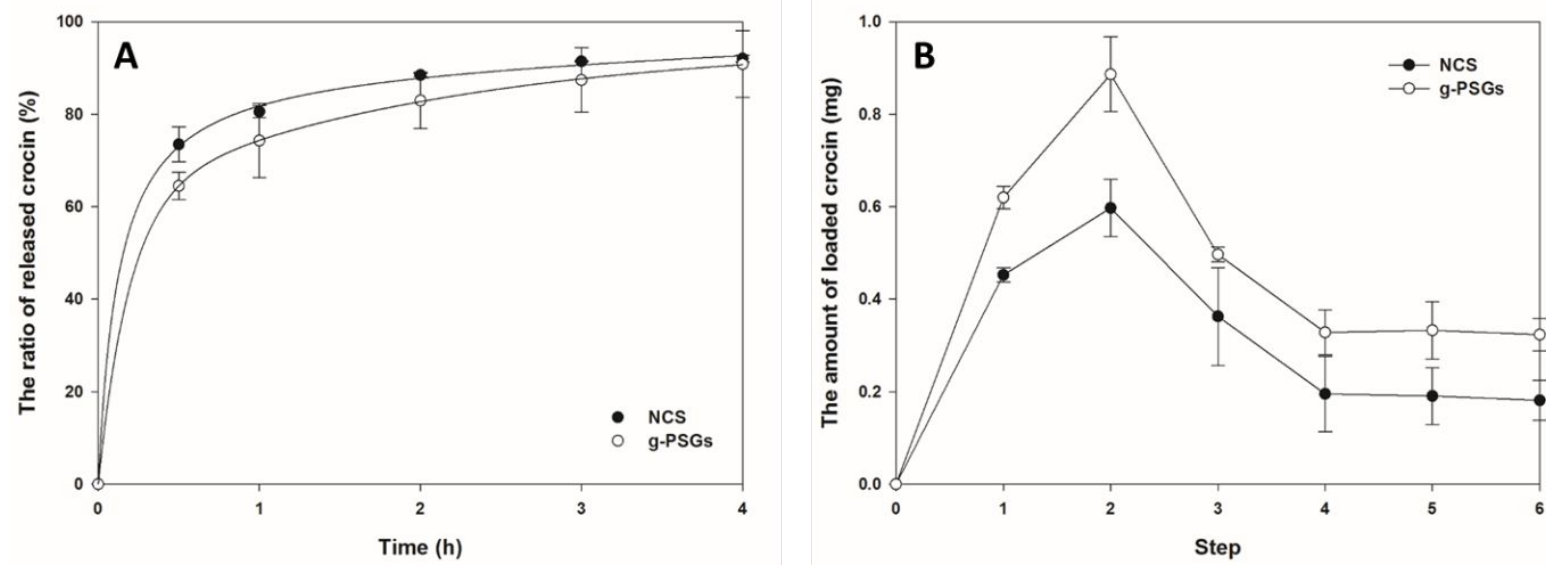

Figure S1. Release patterns of the loaded crocin from the porous starch granules produced by glucoamylase (g-PSGs) and normal corn starch (NCS) by the immersion treatment in aqueous solution over soaking time (A). Preservative effect by the g-PSGs and NCS on the loaded crocin due to the formation of the $\alpha$-glucan coating via elongation reaction by $N p A S$ in each step (B) (0-1, loading step; 1-2, surface coating step; 2-3, washing step; 3-6, releasing step over soaking time). 